\title{
Drying Mechanisms in Plasticized Latex Films. Role of Horizontal Drying Fronts
}

\section{Supporting information}

\author{
V. Divry, A. Gromer, M. Nassar, C. Lambourg, D. Collin, Y. Holl \\ CNRS-ICS \& Université de Strasbourg \\ 23, rue du Loess BP 84047 \\ 67034 Strasbourg Cedex 2 \\ France
}

Drying latex

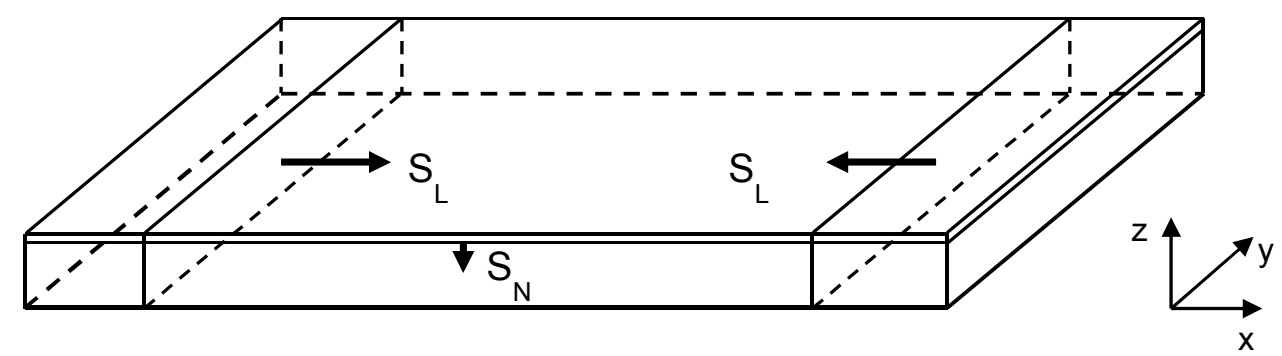

Figure S1. Schematic of a drying latex showing normal (vertical, $\mathrm{z}$ direction) and lateral (horizontal, $x$ direction) drying fronts moving at speeds $S_{N}$ and $S_{L}$, respectively. $S_{L}>S_{N}$. For clarity, lateral drying fronts in the y direction are not represented but also exist.

\section{Decrease of Debye length during drying}

For colloidal dispersions in electrolyte solutions, the Debye length is defined as the distance at which charges are electrically screened. The Debye length is expressed as a function of the ionic strength of the solution FI, the relative permeability of the solvent $\varepsilon_{d}$, the Boltzmann constant $k_{B}$, the temperature $\mathrm{T}$, the elementary charge $e$ and the Avogadro constant $N_{A}$ :

$$
\kappa^{-1}=\sqrt{\frac{\varepsilon_{d} k_{B} T}{2 N_{A} e^{2} F I}}
$$


With the ionic strength $F I=\frac{1}{2} \sum_{i} c_{i} z_{i}^{2}$, where $c_{\mathrm{i}}$ is the molar concentration of ion $\mathrm{i}$ (expressed here in $\mathrm{mol} / \mathrm{m}^{3}$ ), $z_{\mathrm{i}}$ is the charge number of that ion, and the sum is taken over all ions in the solution

In the latex the Debye length is calculated knowing the concentration of sodium hydroxide added to reach a $\mathrm{pH}$ of 10.0. As the latex dries, the ionic strength increases (the ions concentrate by water evaporation), decreasing the Debye length. Figure S1 illustrate this decrease for the latex 200-1 and 200-1+10\%Tex.

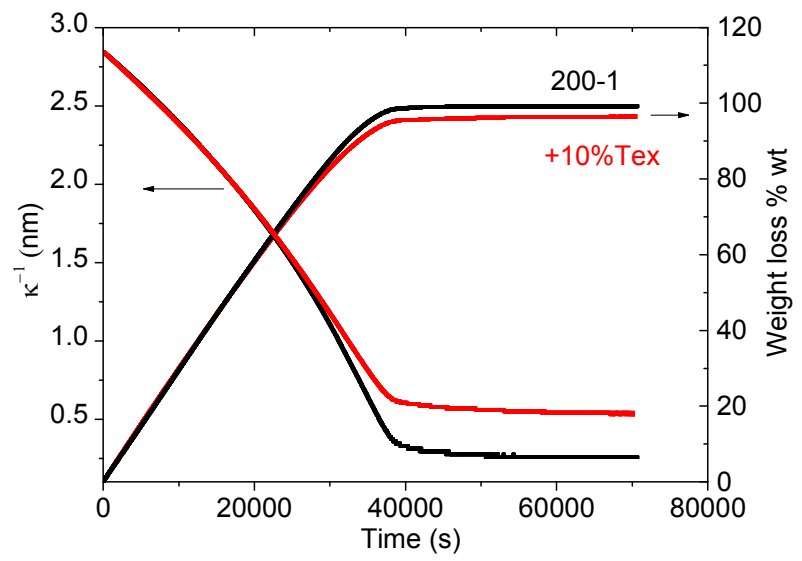

Figure S2: Debye length decrease during drying and Weight loss (in percent of initial weight of water plus Tex) versus time for latexes 200-1 and 200-1+10\%Tex. Drying conditions: $23 \pm 2{ }^{\circ} \mathrm{C}, 75 \pm 2 \% \mathrm{RH}$, still air. Initial film thickness: $770 \mu \mathrm{m}$

Time-lapse videos of 200-1 and 200-1+10\%Tex latex drying:

The drying conditions are $75 \pm 2 \% \mathrm{RH}$ and $23 \pm 2^{\circ} \mathrm{C}$ in still air. The initial film thickness is 770 $\mu \mathrm{m}$. Views of the latexes during drying were recorded every 80 s with a time-lapse camera. The time-lapse videos show 25 frames per second, they are thus 2000 times accelerated.

Links to the videos:

\section{Horizontal drying fronts: comparison with RR model}

Here we summarize the main features of the Routh and Russel models. ${ }^{1,2}$ A semiinfinite film is considered extending in the direction of $x>0$, with $x=0$ corresponding to the film's edge. The film is divided into a wet gel domain, which extends from $x=0$ up to $x=x f$, the position of the particle front, and a fluid domain, for $x>x f$. Vertical inhomogeneities in the particle volume fraction are neglected. Using the Navier-Stokes equation and the lubrication approximation, equations for particle and fluid conservation can be derived in the fluid and wet gel domains (see Table S1).

Table S1: Equations for particle and fluid conservation derived in the fluid and wet gel domains.

\begin{tabular}{|l|l|l|}
\hline & Gel domain & Fluid domain \\
\hline
\end{tabular}




\begin{tabular}{|c|l|l|}
\hline Particle conservation & $\frac{\partial \bar{h} \phi}{\partial \bar{t}}=0$ & $\frac{\partial \bar{h} \phi}{\partial \bar{t}}+\frac{\partial \phi \bar{h} \overline{u_{x}}}{\partial \bar{x}}=0$ \\
\hline Fluid conservation & $1+\frac{\partial \bar{h}(1-\phi)}{\partial \bar{t}}+\frac{\partial \bar{h} \bar{u}_{x}(1-\phi)}{\partial \bar{x}}=0$ & $1+\frac{\partial \bar{h}}{\partial \bar{t}}+\frac{\partial \bar{h} u_{x}}{\partial \bar{x}}=0$ \\
\hline Fluid velocity & $u_{x}=-\frac{k_{p}(\phi)}{\mu} \frac{d p}{d x}$ & $\bar{u}_{x}=\overline{h^{2}} \frac{\partial^{3} \bar{h}}{\partial \bar{x}^{3}}$ \\
\hline
\end{tabular}

In these equations, the film height is scaled on the initial height: $\mathrm{H}_{0}$, time is scaled on a characteristic time: $\frac{H_{O}}{\dot{E}}$, and horizontal distances are scaled on the capillary length:

$L=H_{0}\left(\frac{\gamma}{3 \eta_{o} \dot{E}}\right)^{1 / 4}$, where $\gamma$ is the surface tension at the air/water interface and $\eta_{o}$ the low shear viscosity of the dispersion. The expression for the capillary length is obtained by balancing the flow due to surface tension with that caused by evaporation. The fluid velocity is calculated using a different expression in the gel and fluid domains (see Table S1). In the gel domain, the velocity is given by the Darcy law, where $k_{p}(\phi)=\frac{2 R_{0}^{2}(1-\phi)^{2}}{75 \phi^{2}}$ is the gel permeability, $R_{0}$ the particle radius, $\mu$ the solvent viscosity and $p$ the solvent pressure. In the fluid domain, the expression used reflects the fact that the flow originates from the Laplace pressure at the film surface.

When considering the case of totally non-deformable particles, the film height in the gel is independent of time and the volume fraction is equal to $\phi_{\max }$ (close packed network). Combining the gel domain equations thus leads to the equation for the pressure: $\frac{\partial}{\partial \bar{x}}\left(\bar{h} \frac{\partial \bar{p}}{\partial \bar{x}}\right)=\frac{1}{\left(1-\phi_{\max }\right)}$, where the pressure is scaled on a characteristic pressure: $P=\frac{\mu L^{2} \dot{E}}{k_{p}\left(\phi_{\max }\right) H_{0}}$. Integrating this equation allows to calculate the pressure and fluid velocity in the gel. The film height, volume fraction and dispersion velocity in the fluid domain can then be calculated by integrating the equations from the fluid domain using the condition that, near the particle front, the velocity of the dispersion must be equal to the velocity of water in the wet gel multiplied by $\left(1-\phi_{\max }\right)$. From the particle volume fraction and dispersion velocity, a particle balance allows to calculate the advancement of the particle front. As the wet gel domain grows in size, the pressure differential across it, $\Delta \bar{p}$, increases until it reaches the maximum achievable capillary pressure due to the curvature of the water / air interface: $\Delta \bar{p}=\frac{p_{\max }}{P}$, with: $p_{\max }=\frac{10 \gamma}{R_{0}} \cdot{ }^{3}$ Then, the pressure becomes insufficient to bring water up to the edge and air enters in the film, i.e., a drying front appears. The advancement of the drying front can be calculated by using the velocity of the solvent at that position.

In the case of deformable particles, the volume fraction in the gel is given by:

$\phi=\frac{\phi_{\max }}{1-\epsilon}$, where $\epsilon$ is the strain (assumed to be in the vertical direction). Combining the gel domain equations leads to a new equation for the pressure:

$\left(\frac{2 R_{0}^{2}}{75 \mu \phi_{\max }^{2}}\right) \frac{\partial}{\partial x}\left(h_{\phi=\phi_{\max }}\left(1-\phi_{\max }-\epsilon\right)^{3} \frac{d p}{d x}\right)=\dot{E}-h_{\phi=\phi_{\max }} \frac{\partial \epsilon}{\partial t}$

where $h_{\phi=\phi_{\max }}$ designates the height at the time of close packing. The pressure in the gel translates into a stress applied at the film surface: $p=-\sigma_{t}$ which is assumed to compress the particle network according to:

$\bar{\sigma}_{t}=-\bar{\lambda} \epsilon \frac{d \epsilon}{d \bar{t}}$ where $\bar{\sigma}_{t}=\frac{28 \sigma_{t} R_{0}}{3 v \phi_{\max } \gamma}$

with $v$ the number of nearest neighbors in the particle network. This stress-strain equation can be derived from the Routh and Russel compaction equation for a particle network, ${ }^{4}$ assuming 
compaction by capillary deformation only (no wet sintering). Combining the equation for the pressure with the stress-strain equation allows to calculate both the pressure, film height and volume fraction. Finally, when the volume fraction in the gel reaches one, it is assumed that an optical clarity front appears.

(1) Routh A.F.; Russel W.B. Horizontal Drying Fronts During Solvent Evaporation From Latex Films. AICHE J. 1998, 44, 2088-2098.

(2) Routh, A.F.; El-Aasser, M.S.; Tang, J.; Russel, W. J. Process Model for Latex Film Formation: Optical Clarity Fronts. Coat. Technol. 2001, 73/916, 41-48.

(3) Brown, G.L. Formation of Films from Polymer Dispersions. J. Polym. Sci. 1956, 22, 423 434.

(4) Routh, A.F.; Russel, W.B. A Process Model for Latex Film Formation: Limiting Regimes for Individual Driving Forces. Langmuir 1999, 15, 7762-7773. 